\title{
Polarimetric plasmonic sensing with bowtie nanoantenna arrays
}

\author{
Jonathan Calderón, Jesús Álvarez, Juan Martinez-Pastor, Daniel Hill \\ Unit of Optoelectronic Materials and Devices, Materials Science Institute, \\ University of Valencia, P.O. Box 22085, 46071 Valencia, Spain
}

\begin{abstract}
We propose a polarimetric plasmonic biosensor based on bowtie nanoantenna array transducers. Through numerical simulations, based on the Finite Element Method (FEM), we study the phase retardation between the components of light polarized parallel and perpendicular to the major axis of the bowties within the arrays. From a design for high volumetric sensitivity at a wavelength of $780 \mathrm{~nm}$, sensitivities $\sim 5 \mathrm{rad} / \mathrm{RIU}$ is obtained, corresponding to a detection limit of $\sim 10^{-7}$ when using a polarimetric readout platform. Similarly, surface sensitivity of the same array is evaluated by simulating the phase retardation changes induced by the coverage of bioreceptors and analytes of the metallic nanostructures.
\end{abstract}

\section{Introduction}

Plasmonic nanostructures are metallic materials patterned at the nanoscale that exhibit interesting optical properties due to the large enhancement and localization of the electromagnetic fields. The interaction between a metallic patterns and light leads to a collective oscillations of the electronic charge density localized at the metal surface, called Localized Surface Plasmon Resonance (LSPR). The energy of these plasmons depends on the size, geometry and composition of the nanostructures as well as on the dielectric environment that surrounds them, subsequently many such structures have been engineered to provide diverse functions such as SERS [1], nanotweezing [2, 3] or, as we report here, biosensing [4, 5]. Although the LSPR sensitivity of non-spherical nanoparticles cannot be described analytically, when they are separated by more than $1 \mathrm{~nm}$ their optical properties arise purely from classic electrodynamical effects [6] and so can be modeled through the use of numerical methods such as Discrete Dipole Approximation (DDA), Finite Element Method (FEM) or Finite Differences in Time Domain (FDTD) [7].

Nanostructured plasmonic antennas hold great potential for the realization of highly sensitive optical biosensors as they present a LSPR that is very sensitive to the refractive index of the surrounding media $[8,9]$. When two nanoparticles are close enough for strong coupling to take place between them, electromagnetic fields of several times that of the incident light arise in their gap [10]. Subsequent refractive index variations in the gap result in significantly greater displacements of resonance peaks compared to non-coupled metal nanostructures. Moreover, array structures can induce collective plasmons that can improve this sensitivity further still [11, 12].

To date, most plasmonic nanoantenna biosensing research was reported to have been done with intensity measurements, although typically these are limited due to high levels of noise, such as that resulting from the instability of laser sources. In photonic biosensing this limitation has routinely been demonstrated to be overcome through measurements of the signal phase when sensitive to refractive index variations in the transducer, through a combination of phase modulation of the light source and a post-measurement Fast Fourier Transform or in-situ phase locked loop measurement $[13,14]$.

Specifically in LSPR, the electron cloud of the metallic surface makes a transition of in- to out-ofphase with respect to the incident EM field that can lead to narrower linewidths of the LSPR features and increased values of phase difference defining the Figure of Merit (FOM) of the system [15]. Furthermore measurements of the phase difference between two orthogonally polarized waves after their interaction with a nanoantenna is independent of intensity, provided 
the signal is distinguished from the noise. In fact, LSPR sensing phase has been shown to improve the sensitivity over that from intensity-based measurements [16].

In this paper we propose an alternative scheme for LSPR phase measurement based biosensing by exploiting the symmetry breaking induced by the geometry of a bowtie nanoantenna (Fig. 1). Specifically, the lack of axial symmetry of these nanoantennas results in normally incident light polarized along and perpendicular to the major axis of the bowtie experiencing different phase retardation upon scattering, whose difference will be strongly dependent on the refractive index changes of the surroundings. Moreover, this approach also permits high tolerance alignment with a biosensing instrument consisting of low cost optical components allowing a robust and economical highly sensitive biosening platform.

To the best of our knowledge a theoretical study of arrays of bowtie plasmonic nanoantennas for phase measurement LSPR sensing, where symmetry breaking is induced by the nanostructure geometry has never been reported. The presented study is based on the use of the Finite Element Method (FEM) to calculate the phase retardation as well as the shift in the extinction plasmon peak as a function of the design parameters of a bowtie antenna array on a $\mathrm{SiO}_{2}$ substrate, as compared to an isolated bowtie. By changing the refractive index of the top cladding of the nanoantenna arrays a bulk sensitivity around 5 radians per refractive index unit $(\mathrm{rad} / \mathrm{RIU})$ was obtained, which results in detection limits of $\sim 10^{-7} \mathrm{RIU}$. Lastly, as analyte capture takes place at the metal surface of the nanoantennas within the first 10-20 nm, the surface sensitivity of the nanoantennas array was studied by simulating a coverage of bioreceptor and analyte layers of given refractive indices and thicknesses.

(a)

(b)

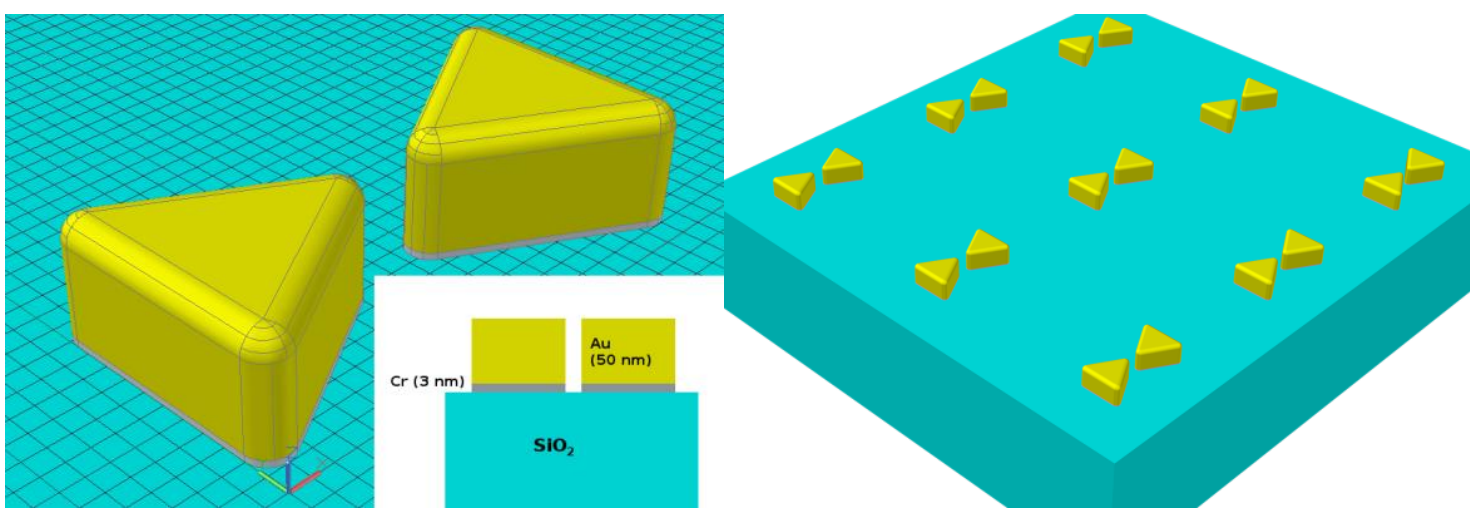

Figure 1: Schematic views of the gold bowtie nanoantenna (a) and the gold bowtie nanoantenna array (b).

\section{Methodology}

The finite element method (FEM) was used to calculate the LSPR characteristics of gold bowtie nanoantennas (Fig. 1), in order to investigate their sensing capabilities. This method allows us to obtain a numerical solution for a structure (in which a set of partial differential equations that characterize its physical behaviour has been defined) by discretizing it into a set of subdomains called "finite elements". The FEM method is a powerful computational tool that has been widely employed to study both the electromagnetic far- and near-fields of metallic nanostructures. In the present work we used COMSOL, a commercial software based on this numerical method.

The refractive index of the substrate (silica) was taken to be 1.51 and the refractive index of the surrounding aqueous media is 1.33 in all simulations. The real and imaginary refractive indices of the chosen plasmonic material, gold, are modeled through an interpolation with splines of the experimental data obtained by Palik et al. [17]. The bowtie nanoantennas were then drawn with a radius of curvature on their tips of $15 \mathrm{~nm}$, so as to avoid divergences induced by extremely spiky tips and to better approximate fabricated nanoantennas [18]. Thereafter a linearly 
polarized electromagnetic plane wave was sent from the surrounding media under normal incidence. A sweep for different wavelengths is performed in order to obtain the plasmonic spectra.

The modeling was done using COMSOL [19], a commercial software based on the FEM; a scattering mode with a background field was introduced as an analytic expression. In the model, two semi-infinite media (water and silica) are used so the background field is defined as the sum of the incident and reflected wave in the first media and the transmitted wave in the second media through the use of the Fresnel equations. This allows us to extract the scattered fields without the need of two simulations, one to calculate numerically the background field and the other to compute the scattered field.

For the simulation of an isolated nanoantenna, we have placed PMLs (Perfect Matched Layers, which are domains with the same refractive index as the surrounding domains at their boundary but with increasing imaginary refractive index when moving away from it) and SBC (Scattering Boundary Conditions, boundaries transparent for scattered waves) around the entire physical domain. These boundary conditions result in an absorption of the EM waves that arrive at the boundaries of our physical domain, preventing unphysical reflections. In order to account for the double-periodic nature of the array simulation, PMLs and SBCs were also placed at the top and the bottom of the unit cell and PECs (Perfect Electric Conditions, which nullify the electric field component perpendicular to the boundary surface) and PMCs (Perfect Magnetic Conditions, which nullify the magnetic field component perpendicular to the boundary surface) on its side walls. The PECs were placed on the two walls perpendicular to the polarization of the normal incident wave and the PMCs on the others, making the side walls act like mirrors, with the effect of a periodic array being obtained [20]. The separation of nanoantennas was equalized to that of the unit cell size.

When simulating the light interaction with the nanostructure the electromagnetic field distribution is derived directly from solving the problem in terms of the fields within the simulated domains and absorption cross-sections are obtained from the integration of the resistive heat generation over the volume of the nanoantenna. Furthermore, the scattering cross-sections are derived from the integration of the Poynting vector (from the consideration of energy conservation [21]):

$$
\sigma_{s c}=\frac{1}{2 \mathrm{I}_{0}} \int\left(\vec{E}_{s c} \times \vec{H}_{s c}\right) d \vec{S}
$$

over a surface that surrounds the nanoantenna, extinction cross-sections are just the sum of the scattering and extinction cross-sections. Extinction efficiency, defined as the fraction between the extinction cross-section and the geometrical cross-section of the nanostructure, instead of scattering cross-section, will be used throughout this paper to allow better comparsion between simulations. The phase is taken as the mean of

$$
\varphi_{i}=\arctan \left(\frac{\operatorname{Im}\left\{E_{i}\right\}}{\operatorname{Re}\left\{E_{i}\right\}}\right)
$$

over a surface parallel to the interface at a distance of one wavelength of the incident wave.

\section{Results and Discussion}

\section{Isolated bowties}

The localized plasmonic near-field of the bowtie nanoantennas, which is very sensitive to the refractive index of its surrounding medium can be tuned by modifying the nanostructure shape. Geometrical parameters such as size, gap distance [22], height [23], bowtie angle [24, 25] and sharpness of the tips $[20,26]$ have a direct influence in the LSPR, changing the spectral position and narrowness of the plasmonic peak in the extinction cross-section. Taking into account the fabrication limitations and aiming for a LSPR peak wavelength around $780 \mathrm{~nm}$, which is a standard wavelength of near IR diode lasers used in Surface Enhanced Raman Scattering (SERS), we fixed the height of the nanoparticles to $50 \mathrm{~nm}$, the length of their sides to $120 \mathrm{~nm}$ and the curvature of the vertices to a $15 \mathrm{~nm}$ sphere radius. The nanoparticles were modeled as 
equilateral triangles and a chrome adhesion layer of $3 \mathrm{~nm}$ of thickness was added under the gold nanoparticles, as can be observed in Fig. 1 (a), which is needed in actual applications for the gold to adhere to the $\mathrm{SiO}_{2}$ substrate. The presence of a metal adhesion layer induces the broadening of the LSPR and diminishes the scattering amplitude [27] and therefore the adhesion layer should be maintained as thin as possible. The choice of the substrate is very important due to the screening effect [28] and so it is convenient to choose a substrate with a refractive index as close as possible to that of the medium that surrounds the bowties.

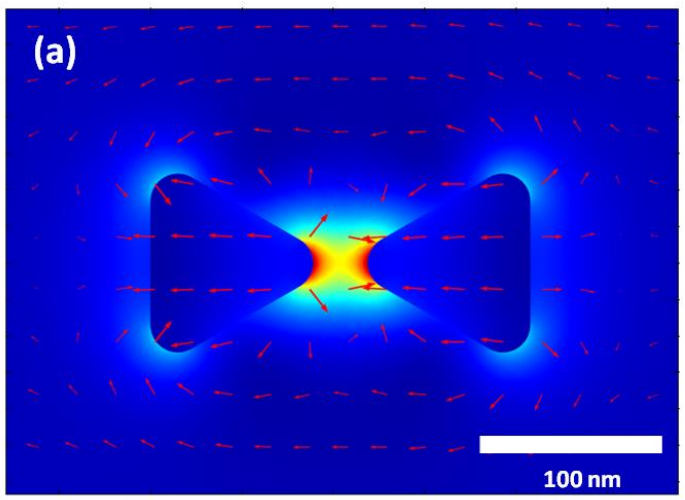

(c)

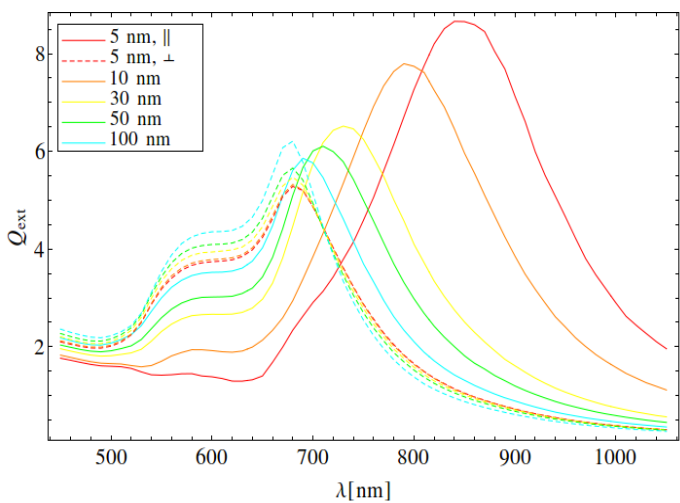

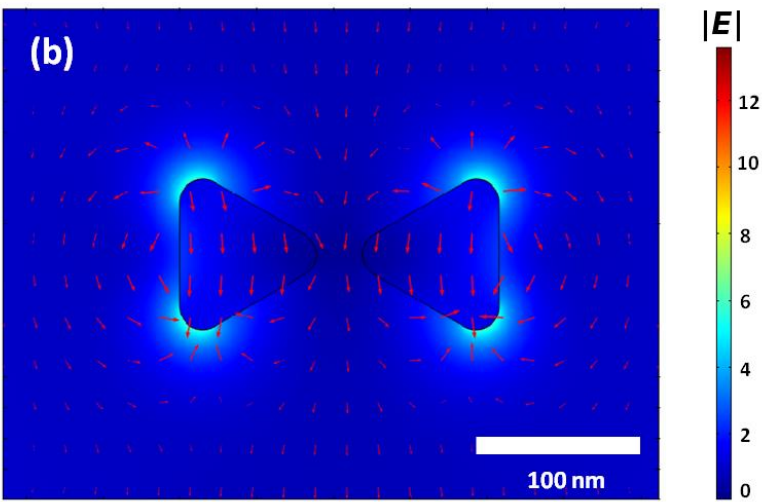

(d)

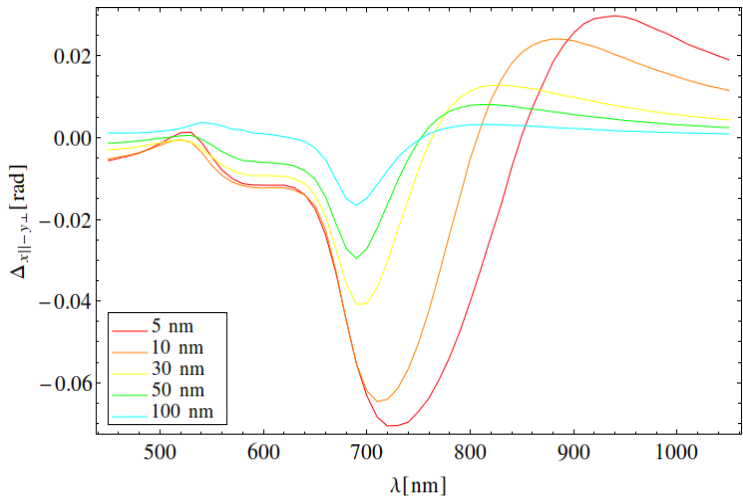

Figure 2: Electric field distribution at the LSPR frequency for incident light polarized along (a) and perpendicular (b) to the major axis of a bowtie nanoantenna with a gap of $30 \mathrm{~nm}$ (c). Extinction spectra of the bowtie nanoantenna for polarization parallel (continuous lines) and perpendicular (dashed lines) to the major axis of the bowtie for different gaps (d) Phase difference spectra for different gaps.

As expected, an incident linearly polarized wave with electric field parallel to the major axis of the bowtie results in a high confinement of electric field within the gap region of the nanoantenna (Fig. 2a), with enhancement factors (|E|/|EO|) up to a factor 100 for the smallest gap simulated $(5 \mathrm{~nm})$. However, if the polarization is perpendicular, the enhancement regions are located around the other tips of the bowtie [Fig. 2 (b)], far from the gap region, and the enhancement factor becomes considerably lower.

We then carried out simulations for various gap lengths, calculating the extinction spectra for both polarization directions in order to study the plasmon peak width and shift [Fig. 2(c)]. Along the direction parallel to the major axis of the bowtie an increasing redshift of the more intense and lower energy plasmon resonance is observed by narrowing the gap, contrary to the perpendicular case,(shorter wavelength plasmon resonance), where the redshift is practically absent. These resonant plasmonic modes at short and long wavelengths are characteristics of the single particle and dimmer of the bowtie, respectively, and calculated extinction crosssection is consistent with extinction efficiency and transmission data for similar nanoparticles modelled elsewhere [22]. Actually, as the length of the gap increases the extinction spectra of the perpendicular and parallel excitations converge, this fact and the absence of a significant redshift in the perpendicular mode can both be explained by the separation of field 
enhancement locations: direct dipolar coupling (which is a near-field effect that decreases as $\left.\sim 1 / r^{3}\right)$ ) between the two particles is very weak. The particles become polarized when the incident light is polarized along the major axis, this induces a charge separation and with it an attractive force that lowers the plasmon energy. The effect diminishes with increasing gap length until the plasmon energy arrives at that of an isolated particle. The broadening of the plasmon for smaller gaps observed in the parallel excitation is another consequence of the dipolar coupling. The maximum shift of the long wavelength (longitudinal) plasmon resonance associated to the bowtie dimmer is found when both prism nanostructures are touching and hence constituting a new single metal nanostructure. By increasing the prism size further, a certain tuning of the longitudinal plasmon mode can be achieved, but the effect of electric field concentration at the bowtie gap will be not very different given the broad wavelength range defined by this mode. The electric field concentration is mainly determined by the reduction of the bowtie gap distance, when the evanescent electric field perpendicular to each metal nanostructure overlaps, as observed in Fig. 2 (a). Accordingly, when the bowtie gap distance is sufficiently small the electric field enhancement increases exponentially.

The phase of the scattered wave is calculated from Equation 2 for both polarizations and the phase difference between them is determined by

$$
\Delta_{\varphi}=\varphi_{x}^{\|}-\varphi_{y}^{\perp}
$$

according to the electric field components that dominate in both polarization modes. Characteristic spectra were then obtained for each gap distance [Fig. 2 (c)] and once again two plasmon resonances are observed. The one at a longer wavelength is that associated with the polarization along the major axis of the bowtie system, for which a noticeable redshift and broadening is observed, as it is also in the extinction spectra.

In the subsequent sections a gap of $30 \mathrm{~nm}$ was chosen for all simulations so that the bowtie nanoantenna may have a good sensitivity whilst still being feasible for fabrication by technologies suitable for production of commercial biosensors [29].

\section{Bowtie array}

If the wavelength of the scattered light is close to both the pitch distances of the array and the LSPR wavelength then a dramatic modification of the extinction cross-section will arise due to a collective coupling of every localized plasmon. When excited by an EM wave at frequency $\omega, a$ dipole radiates a scattered wave proportionally to its dipole moment and so the net field at every dipole is therefore the sum of the incident field plus the radiation of all the others dipoles, which leads to a system of coupled equations. Assuming an infinite array, the effective polarizability, a complex variable, is:

$$
\alpha^{*}=\frac{1}{1 / \alpha-S^{\prime}}
$$

where $\underline{S^{\prime}}$ is an array factor that encompasses the influence of the other dipoles and is therefore only dependent of geometrical parameters. In the case of arrays with equal pitch of nanoparticles in both orthogonal directions in the plane, or square arrays, and normal incidence we have [30]:

$$
S^{\prime}=\sum_{\text {dipoles }} e^{i k r}\left[\frac{(1-i k r)\left(3 \cos ^{2} \theta-1\right)}{r^{3}}+\frac{k^{2} \sin ^{2} \theta}{r}\right]
$$

The first term describes the near-field dipolar coupling that decreases with $1 / r^{3}$ and the second term represents the radiative coupling that decreases with $1 / r$ and, hence, is more strongly affected by the array geometry. The poles of $\alpha^{*}$ define the resultant resonances from the bowtie coupling, which are a function of the physical properties of the nanoparticles and the geometry of the array. When the imaginary part of $S^{\prime}$ is negative, a partial cancellation of the radiative damping appears, which causes a narrowing of the resonance.

By virtue of the Optical Theorem we know that the extinction cross-section is proportional to the polarizability:

$$
\sigma_{e x t} \propto k \operatorname{Im}\{\alpha\}
$$

A peak in the extinction curve is obtained when the real part of $1 / \alpha-S^{\prime}$ vanishes in Equation 4. 
Therefore, by adjusting the pitch distances of the array, a narrowing of the resonance can be achieved through radiative coupling. This model, based on the Dipole Array Approximation (DAA), works very well when the nanoparticles of the arrray are spheres and ellipsoids and can be easily numerically calculated [11, 12, 31]. However, when we consider triangular prisms like the present case, the expression of the polarizabillity becomes extremely complex and numerical simulations based on methods such as FEM, DDA or FDTD are demanded [32].

Once the size, gap and height of the bowties were decided, FEM simulations for different values of horizontal and vertical pitch periods (Dx and Dy) were run, a sample of these simulations can be found in Fig. 3 (b), where can be observed how the lineshape of the LSPR spectra can be tuned by varying the pitch period of the array. The main criterion was to obtain the narrowest plasmonic peak centered at around $780 \mathrm{~nm}$ for the parallel mode plasmon, and hence, the best sensitivity in intensity at this wavelength. The decrease of the horizontal pitch at a fixed vertical pitch shows a redshift and broadening of the plasmon [Fig. 3 (a)], analogous to the behaviour of the plasmons in the isolated bowties upon decreasing the gap distance. Tha variation of the vertical pitch at a fixed horizontal pitch it's more complex and suggests that different modes arises from the vertical coupling [Fig. 3 (b)]. The optimum pitches were found to be Dx $=525 \mathrm{~nm}$ and Dy $=525 \mathrm{~nm}$ and so this array [Fig. 3 (c)] was used in all of the sensing simulations reported in sections 3.1 and 3.2. Although this optimization of geometrical parameters for intensity sensing does not ensure that it is also optimized for polarimetric sensing, initial optimization for polarimetric sensing shows that they provide a good initial approximation. It is therefore expected that an optimization of the pitches based directly on the phase difference could lead to even better results than those shown in the next section.

(a)

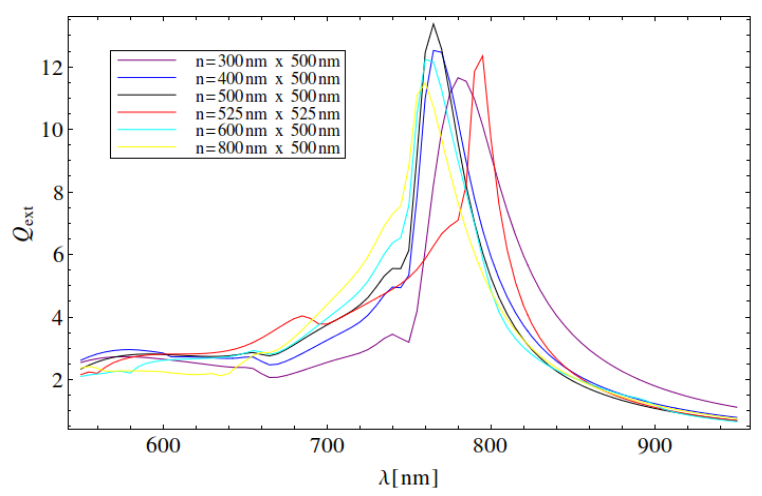

(c)

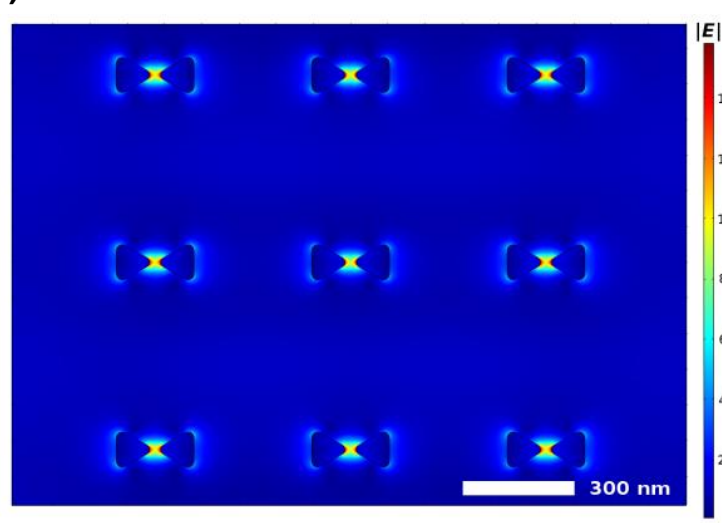

(b)

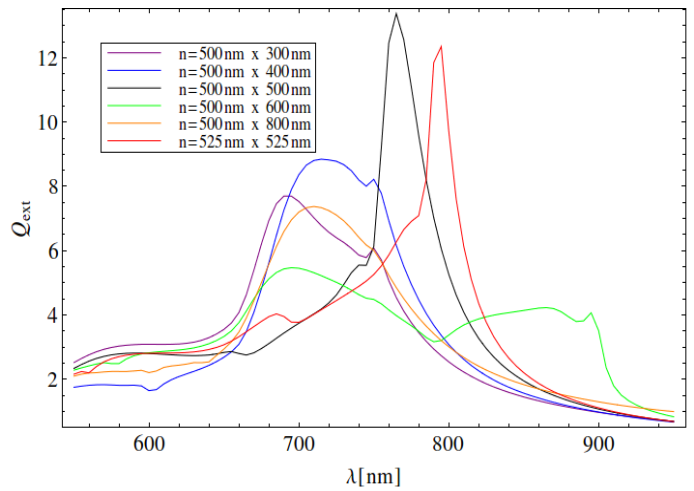

Figure 3: Extinction efficiencies obtained from the simulation of arrays under parallel polarization varying the horizontal pitch distance (a) and the vertical pitch distance (b). (c) Electric field distribution at the LSPR frequency for the chosen bowtie array (both pitch periodes of $525 \mathrm{~nm}$, gap of $30 \mathrm{~nm}$ ).

\subsection{Bulk refractive index sensing}

In reflectance and transmittance measurements the intensity signal is proportional to the extinction cross section, and so by studying the variation of the extinction spectra with respect to the refractive index we can obtain the sensitivity and thereafter detection limit of the sensing 
system based on the phase retardation measurement. We therefore performed simulations for different values of the refractive index of the medium occupying the half space above the bowtie array for both EM field polarizations; the results were then compared with those for an isolated nanoantenna (Fig. 4).

(a)

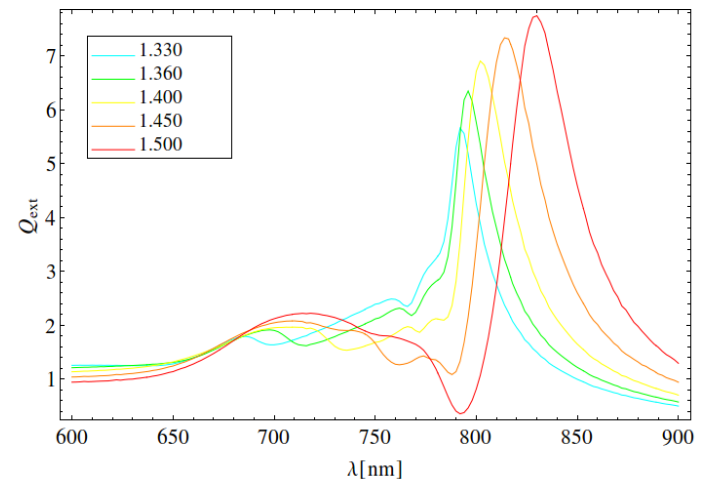

(b)

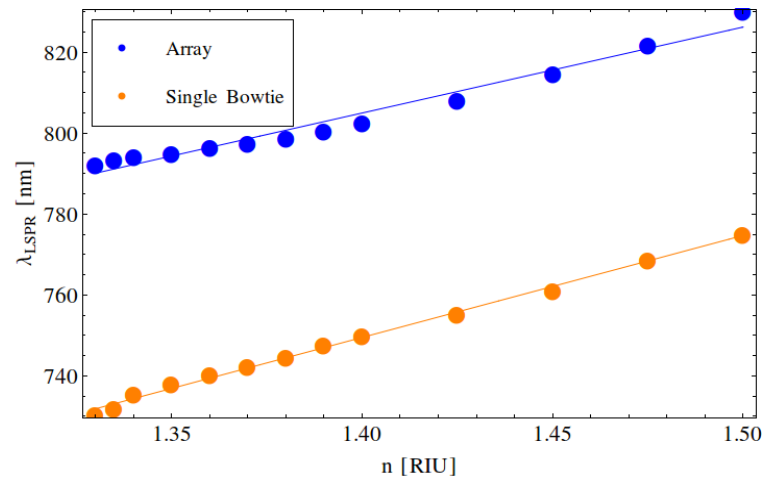

Figure 4: (a) Extinction efficiency for different refractive index values of the surrounding media for the array (b) A comparison of extinction efficiency LSPR primary peak wavelengths for the array and the single bowtie under parallel polarization.

We define the sensitivity of our system as the change in the wavelength of the LSPR per the variation of the refractive index as:

$$
S=\frac{\partial \lambda_{L P S R}[\mathrm{~nm}]}{\partial n[R I U]}
$$

When plotting the LSPR peak wavelengths from Fig. 4 (a), against the refractive index of the surrounding medium we observe in Fig. 4 (b) that the slope is slightly greater for the single bowtie than for the array ( $231 \mathrm{~nm} / \mathrm{RIU}$ against $246 \mathrm{~nm} / \mathrm{RIU})$ and thus a better sensitivity will be obtained if considering the LSPR redshift. However when we compare Fig. 2 (c) with Fig. 4 (a) we can see that the peaks are much narrower for the array, which would lead to an improvement in the limit of detection by one order of magnitude at the wavelength where the LSPR derivative is greater, with FOMs differing by one order of magnitude, too; that is, considering the variation of the intensity at a fixed wavelength instead of the shift of the plasmon peak.

A similar study was then done this time using the phase difference between the two polarization excitations:

(a)

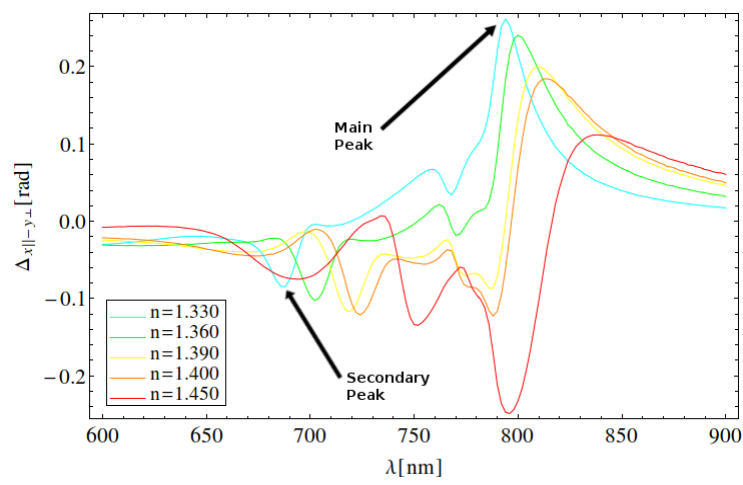

(b)

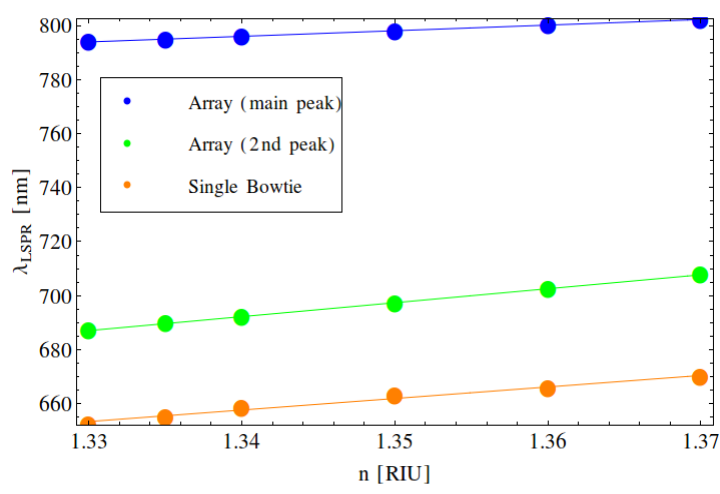

Figure 5: (a) Phase difference spectra for different refractive index values of the surrounding media for the bowtie array. (b) A comparison of phase difference LSPR peak wavelengths for the secondary peak and the main peak. 
We define the phase sensitivity as the change in phase retardation over the variation of the refractive index [33]:

$$
S_{\text {phase }}=\frac{\partial \Delta[\mathrm{rad}]}{\partial n[R I U]}
$$

and the Detection Limit (DL) can be defined as the resolution of the phase measurement system (sigma) divided by the phase sensitivity of our nanostructure (Equation 8):

$$
D L=\frac{\sigma[\mathrm{rad}]}{S_{\text {phase }}[\mathrm{rad} / \mathrm{RIU}]}
$$

The resolution of the setup was tested by taking multiple measurements of a quartz sample with a known birefringence. The measured phase difference values had a Gaussian distribution with a standard deviation equal to $10^{-7} \mathrm{rad}$ [34]. By adopting the convention that the resolution is given by their standard deviation, we obtain the resolution of our measurement system as $10^{-7}$.

The best phase sensitivities obtained were $0.43 \mathrm{rad} / \mathrm{RIU}$ for the isolated bowties at a wavelength of $720 \mathrm{~nm}$ and $4.75 \mathrm{rad} / \mathrm{RIU}$ for the array bowties at a wavelength of $790 \mathrm{~nm}$, which is not surprising given the sharper profile of the LSPR features in Fig. 5 (a), as compared to those in Fig. 2 (c).

(a)

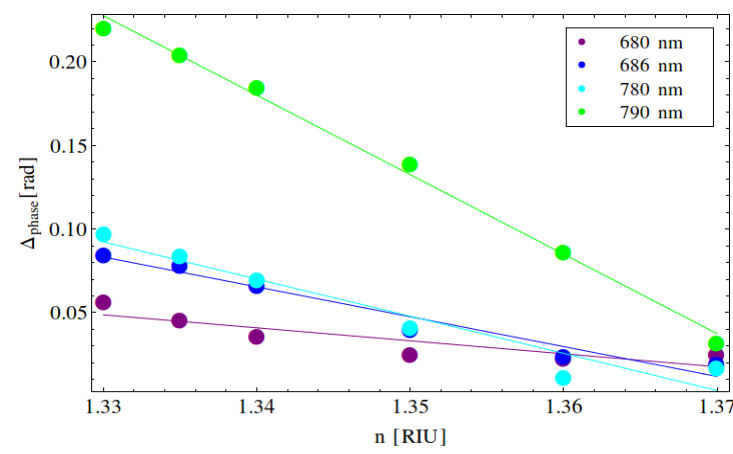

(b)

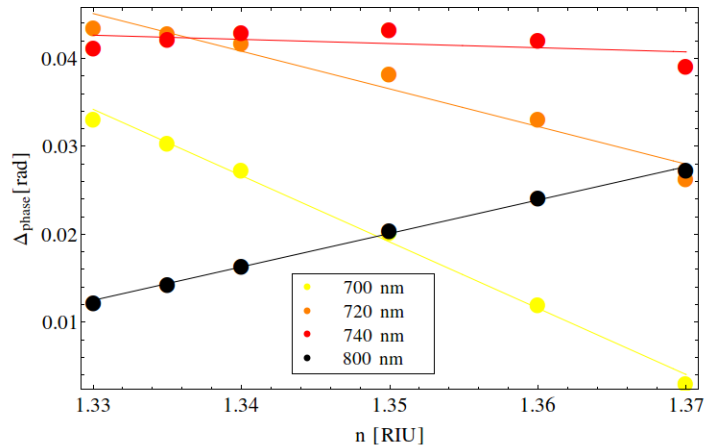

Figure 6: Phase sensitivity fits at different wavelengths for the array (a) and the single bowtie (b)

The redshift rate of the secondary LSPR peak is far greater than that of the main peak, even this one is narrower and therefore can provide a lower detection limit: below $10^{-7} \mathrm{RIU}$ for the array and below $10^{-6}$ for the isolated bowtie. The difference of DLs between isolated bowties and bowtie arrays are due again from the broader plasmon resonances present in the isolated bowties.

\subsection{Surface sensing}

For many bioassays on a transducer the analyte capture would take place within the first 10-20 $\mathrm{nm}$ from the surface of the nanoantennas, where the electromagnetic field is enhanced, an so the surface sensitivity of the nanoantennas array was studied by simulating a coverage of $5 \mathrm{~nm}$ thick bioreceptor and analyte layers on them under parallel and perpendicular polarization. The first layer, the bioreceptor, was defined by a constant refractive index of 1.39 , which is a suitable value for a protein layer $[35,36]$, and the second, the analyte, was chosen as a variable refractive index in order to simulate various surface concentrations. The biomolecular coating leads to the redshift of the resonance wavelength. This can be explained by the increase of the effective optical path caused by the larger refractive indices of the coating [23]. 


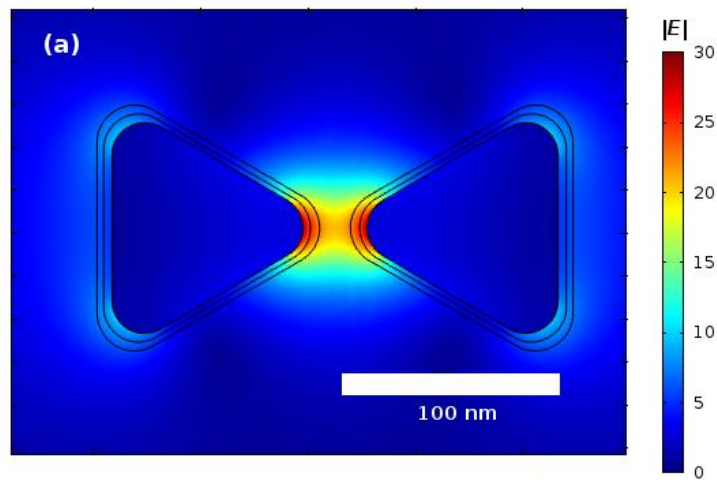

(c)

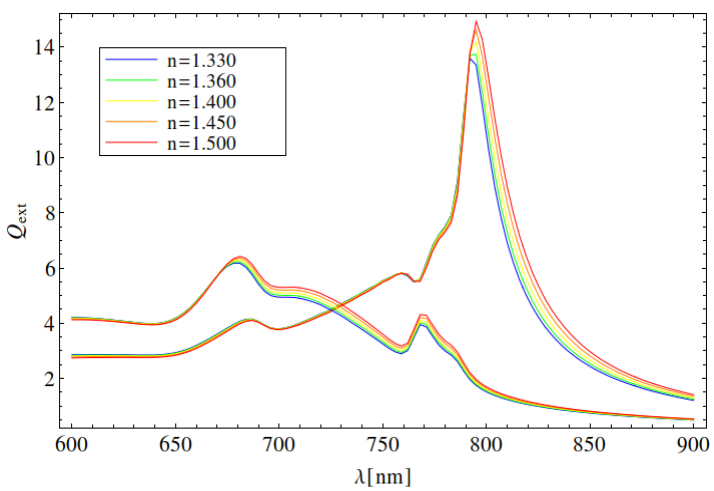

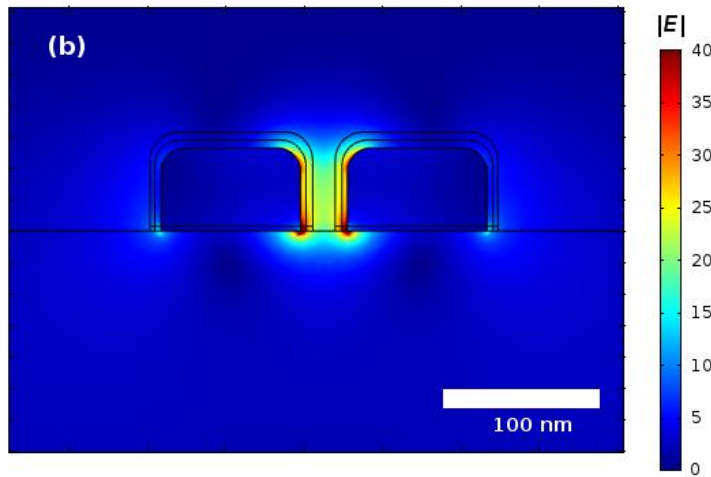

(d)

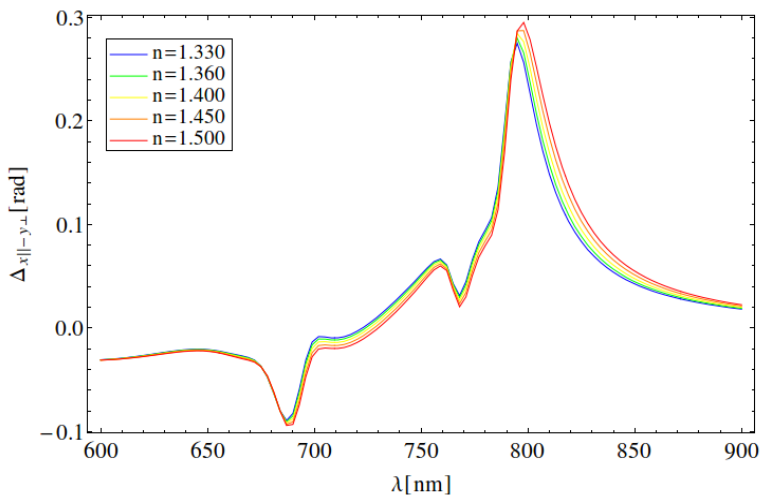

Figure 7: Cross section (a) and plane view (b) of the electric field surrounding the dual biomolecule layer covered nanoantenna for parallel polarization. (c) Extinction spectra of the covered bowtie array for different refractive indexes (d) Phase difference spectra of the covered bowtie array for different refractive indexes of the outer biomolecular layer.

The DLs obtained for the phase measurement are less than $10^{-5}$, one and two orders of magnitude greater than the volumetric DLs of the single bowtie and array, respectively, which is consistent with other studies of surface detection limits, involving metallic nanogratings for instance [37]. This can be explained by the smaller effective volume of analyte with respect to the bulk scenario where all the surrounding media and, more importantly, the entire cavity between the tips, where the field enhancement is greater, is full of the substance we want to sense.

\section{Conclusions}

Simulations based on the Finite Element Method were performed to optimize bowtie array designs in order to find the most suitable plasmonic peak for biosensing. From the optimal design, the sensitivity was then determined by simulating the phase retardation for both perpendicular polarizations of the incident plane wave upon varying the refractive index of the surrounding aqueous medium. A similar analysis for surface sensitivity was then performed through the addition of bound layers of bioreceptors and analytes, for a variable analyte RI. Volumetric detection limits below $10^{-7}$ are obtained for the gold bowtie array based polarimetric sensor comparing them favourably with state of the art plasmonic sensors [38], while surface detection limits were found to be two orders of magnitude higher. Moreover, within a future study an optimization of the pitch between bowties based directly on the phase difference could lead to lower limits still.

\section{Acknowledgments}

This work was supported through the Generalitat Valenciana and Spanish MINECO under Grants Nos. PROMETEO/2009/074 and TEC2011-29120-C05-01, respectively. Thanks are also given to the FORTEZA program of the Generalitat Valenciana. 


\section{References}

[1] Nahla A. Hatab, Chun-Hway Hsueh, Abigail L. Gaddis, Scott T. Retterer, Jia-Han Li, Gyula Eres, Zhenyu Zhang, and Baohua Gu, Free-Standing Optical Gold Bowtie Nanoantenna with Variable Gap Size for Enhanced Raman Spectroscopy, DOI: 10.1021/nl102963g | Nano Lett. 2010, 10, 4952-4955

[2] Brian J. Roxworthy, Kaspar D. Ko, Anil Kumar, Kin Hung Fung, Edmond K. C. Chow, Gang Logan Liu, Nicholas X. Fang, and Kimani C. Toussaint, Jr. , Application of Plasmonic Bowtie Nanoantenna Arrays for Optical , Trapping, Stacking, and Sorting, DOI: 10.1021/nl203811q | Nano Lett. 2012, 12, 796-801

[3] Brian J. Roxworthy and Kimani C. Toussaint, Jr., Plasmonic nanotweezers: strong influence of adhesion layer and nanostructure orientation on trapping performance, doi: 10.1364/OE.20.009591 | Vol. 20, No. 9 / Optics Express 9591 (2012)

[4] Jeffrey N. Anker, W. Paige Hall, Olga Lyandres, Nilam C. Shah, Jing Zhao and Richard P. Van Duyne, Biosensing with plasmonic nanosensors, DOI: 10.1038/nmat2162 | Nature Materials 7, 442 - 453 (2008)

[5] Srdjan S. Acimović, Mark P. Kreuzer, María U. González and Romain Quidant, Plasmon Near-Field Coupling in Metal Dimers as a Step towards Single-Molecule Sensing, DOI: 10.1021/nn900102j | AcsNano Vol. 3, No. 5, 1231-1237 (2009)

[6] Ruben Esteban, Andrei G. Borisov, Peter Nordlander \& Javier Aizpurua, Bridging quantum and classical plasmonics with a quantum-corrected model, nature communications, DOI: 10.1038/ncomms1806 | Nature Communicationes 3, No. 825 1-9 (2012)

[7] Zhao, J.; Pinchuk, A. O.; Mcmahon, J. M.; Li, S. Z.; Ausman, L. K.; Atkinson, A. L.; Schatz, G. C., Methods for describing the electromagnetic properties of silver and gold nanoparticles, DOI: 10.1021/ar800028j | Acc. Chem. Res. 2008, 41, 1710.

[8] Kathryn M. Mayer and Jason H. Hafner, Localized Surface Plasmon Resonance Sensors, Chem. Rev. 2011, 111, 3828-3857

[9] Takumi Sannomiya, Christian Hafner, and Janos Voros, In situ Sensing of Single Binding Events by Localized Surface Plasmon Resonance, DOI: 10.1021/nl802317d | Nano Lett., Vol. 8, No. 10,2008

[10] Arvind Sundaramurthy, K. B. Crozier, G. S. Kino, D. P. Fromm, P. J. Schuck and W. E. Moerner, Field enhancement and gap-dependent resonance in a system of two opposing tip-totip Au nanotriangles, Physical Review B 72, 165409 (2005)

[11] Ronen Adato, Ahmet Ali Yanik, Chih-Hui Wu, Gennady Shvets and Hatice Altug, Radiative engineering of plasmon lifetimes in embedded nanoantenna arrays, 1 March 2010 / Vol. 18, No. 5 / Optics Express 4526

[12] Shengli Zou, Nicolas Janel, and George C. Schatz, Silver nanoparticle array structures that produce remarkably narrow plasmon lineshapes, DOI: 10.1063/1.1760740 | J. Chem. Phys. 120, 10871 (2004)

[13] J. Álvarez, C. Serrano, D. Hill, J. Martínez-Pastor, Real-time polarimetric optical sensor using macroporous alumina membranes, Optic Letters 38, 7, (2013), pp 1058-1060

[14] Stefania Dante, Daphné Duval, Borja Sepúlveda, Ana Belen González-Guerrero, José Ramón Sendra and Laura M. Lechuga, All-optical phase modulation for integrated interferometric biosensors, 26 March 2012 / Vol. 20, No. 7 / Optics Express 7195

[15] Kristof Lodewijks, Willem Van Roy, Gustaaf Borghs, Liesbet Lagae and Pol Van Dorpe, Boosting the Figure Of Merit of LSPR-based refractive index sensing by phase-sensitive measurements, DOI: 10.1021/nl300044a | Nano Lett. 2012, 12 (3), 1655-1659

[16] Chung-Tien Li, How-foo Chen, leng-Wai Un, Hsin-Cheng Lee and Ta-Jen Yen, Study of optical phase transduction on localized surface plasmon resonance for ultrasensitive detection, 30 January 2012 / Vol. 20, No. 3 / Optics Express 3251

[17] E. D. Palik, Handbook of Optical Constants of Solids (Academic Press, 1997).

[18] P. J. Schuck, D. P. Fromm, A. Sundaramurthy, G. S. Kino and W. E. Moerner, Improving the Mismatch between Light and Nanoscale Objects with Gold Bowtie Nanoantennas, PRL 94, 017402 (2005)

[19] COMSOL MULTIPHYSICS, Comsol Inc. (http://www.comsol.com)

[20] Alexander V. Kildishev, Joshua D. Borneman, Kuo-Ping Chen and Vladimir P. Drachev, Numerical Modeling of Plasmonic Nanoantennas with Realistic 3D Roughness and Distortion, DOI: 10.3390/s110707178 / Sensors 2011, 11, 7178-7187

[21] John David Jackson, Classical Electrodynamics (Third Edition)

[22] Stephanie Dodson, Mohamed Haggui, Renaud Bachelot, Jérôme Plain, Shuzhou Li and 
Qihua Xiong, Optimizing Electromagnetic Hotspots in Plasmonic Bowtie Nanoantennae, DOI: 10.1021/jz302018x | J. Phys. Chem. Lett. 2013, 4, 496-501

[23] Tzy-Rong Lin, Shu-Wei Chang, Shun Lien Chuang, Zhaoyu Zhang and P. James Schuck, Coating effect on optical resonance of plasmonic nanobowtie antenna, Applied Physics Letters 97, 063106 (2010)

[24] Wei Ding, Renaud Bachelot, Sergei Kostcheev, Pascal Royer and Roch Espiau de Lamaestre, Surface plasmon resonances in silver Bowtie nanoantennas with varied bow angles, DOI: 10.1063/1.3524504 | Journal of Applied Physics 108, 124314 (2010)

[25] Gang Bi, Li Wang, Li Ling, Yukie Yokota, Yoshiaki Nishijima, Kosei Ueno, Hiroaki Misawa, Jianrong Qiu, Optical properties of gold nano-bowtie structures, DOI: 10.1016/j.optcom.2012.12.080 | Optics Communications 294 (2013) 213-217

[26] Hongming Shen, Guowei Lu, Tianyue Zhang, Jie Liu, Ying Gu, Pascal Perriat, Matteo Martini, Olivier Tillement and Qihuang Gong, Shape effect on a single-nanoparticle based plasmonic nanosensor, Nanotechnology 24 (2013) 285502

[27] Xiaojin Jiao, Jeremy Goeckeritz, Steve Blair, Mark Oldham, Localization of Near-Field Resonances in Bowtie Antennae: Influence of Adhesion Layers, DOI: 10.1007/s11468-0089075-x | Plasmonics (2009) 4:37-50

[28] Mark W. Knight, Yanpeng Wu, J. Britt Lassiter, Peter Nordlander and Naomi J. Halas, Substrates Matter: Influence of an Adjacent Dielectric on an Individual Plasmonic Nanoparticle, DOI: 10.1021/nl900945q | Nano Lett. 9, 5, 2009, 2188-2192

[29] Nathan C Lindquist, Prashant Nagpal, Kevin M McPeak, David J Norris and Sang-Hyun Oh, doi: 10.1088/0034-4885/75/3/036501| Engineering metallic nanostructures for plasmonics and nanophotonics, | Rep. Prog. Phys. 75 (2012) 036501 (61pp)

[30] Baptiste Auguie and William L. Barnes, Collective Resonances in Gold Nanoparticle Arrays, Phys. Rev. Lett. 101, 143902 (2008)

[31] Daniel Weber, Pablo Albella, Pablo Alonso-González, Frank Neubrech, Han Gui, Tadaaki Nagao, Rainer Hillenbrand, Javier Aizpurua and Annemarie Pucci, Longitudinal and transverse coupling in infrared gold nanoantenna arrays: long range versus short range interaction regimes, 1 August 2011 / Vol. 19, No. 16 / Optics Express 15047

[32] Chun-Hway Hsueh, Chih-Hong Lin, Jia-Han Li, Nahla A. Hatab and Baohua Gu, Resonance modes, cavity field enhancements and long-range collective photonic effects in periodic bowtie nanostructures, 26 September 2011 / Vol. 19, No. 20 / Optics Express 19660

[33] Jan Becker, Andreas Trügler, Arpad Jakab, Ulrich Hohenester and Carsten Sönnichsen, The Optimal Aspect Ratio of Gold Nanorods for Plasmonic Bio-sensing, DOI:10.1007/s11468010-9130-2 | Plasmonics (2010) 5:161-167

[34] J. Álvarez, N. Kumar, P. Bettotti, D. Hill, J. Martínez-Pastor, Phase-Sensitive Detection for Optical Sensing With Porous Silicon, IEEE Photonics Journal, Volume 4, Number 3, June 2012 [35] Graham H. Cross, Andrew A. Reeves, Stuart Brand, Jonathan F. Popplewell, Louise L. Peel, Marcus J. Swann, Neville J. Freeman, A new quantitative optical biosensor for protein characterisation, Biosensors and Bioelectronics 19 (2003) 383-390

[36] Janos Boros, The density and refractive index of adsorbing protein layers, Biophysical Journal Volume 87 July 2004 553-561

[37] Silvio Savoia, Armando Ricciardi, Alessio Crescitelli, Carmine Granata, Emanuela Esposito, Vincenzo Galdi and Andrea Cusano, Surface sensitivity of Rayleigh anomalies in metallic nanogratings, DOI:10.1364/OE.21.023531 | 7 October 2013 / Vol. 21, No. 20 / Optics Express 23532

[38] Ian M. White and Xudong Fan, On the performance quantification of resonant refractive 